\title{
Examining vulnerability to involuntary memories in schizophrenia comorbid with post-traumatic stress disorder
}

Article

Accepted Version

Jones, V. and Steel, C. (2014) Examining vulnerability to involuntary memories in schizophrenia comorbid with posttraumatic stress disorder. Schizophrenia Research, 152 (2). pp. 487-489. ISSN 0920-9964 doi:

https://doi.org/10.1016/j.schres.2013.11.034 Available at https://centaur.reading.ac.uk/36349/

It is advisable to refer to the publisher's version if you intend to cite from the work. See Guidance on citing.

To link to this article DOI: http://dx.doi.org/10.1016/j.schres.2013.11.034

Publisher: Elsevier

All outputs in CentAUR are protected by Intellectual Property Rights law, including copyright law. Copyright and IPR is retained by the creators or other copyright holders. Terms and conditions for use of this material are defined in the End User Agreement.

www.reading.ac.uk/centaur 
Central Archive at the University of Reading

Reading's research outputs online 


\title{
Schizophrenia Research Short Report
}

\section{Examining vulnerability to involuntary memories in schizophrenia comorbid with post-traumatic stress disorder}

Vanessa Jones and Craig Steel

\begin{abstract}
The current study explored whether individuals diagnosed with schizophrenia and a high level of PTSD symptoms experience more frequent neutral intrusive memories than individuals diagnosed with schizophrenia with low level PTSD symptoms. Results supported a vulnerability to neutral intrusive memories within the comorbid group, which did not seem to be related to psychotic symptom severity. It is possible that a subgroup of psychotic individuals' process information in a manner that make them susceptible to frequent intrusive memories, characteristic of a PTSD presentation. A longitudinal study is required to specify the development of this vulnerability so as to inform future interventions.
\end{abstract}

\section{Keywords}

Schizophrenia; Post-traumatic Stress Disorder; intrusions; involuntary memory; cognitive model 


\section{Introduction}

Whilst intrusive memories occur in many psychiatric disorders including social phobia, obsessive compulsive disorder and bipolar disorder (Gregory et al.; Hackmann et al.; Speckens et al.), intrusive memories of past distressing events are the 'hallmark' symptom of posttraumatic stress disorder (PTSD). However, recent research suggests that the prevalence of traumatic life events within individuals diagnosed with schizophrenia is $94 \%$ and that $15 \%-53 \%$ (Kilcommons and Morrison, 2005) suffer from comorbid PTSD. The prognosis of this comorbid group is poor and the cost of treatment is consequently high (Spauwen et al., 2006). Our current understanding of the psychological impact of trauma on people diagnosed with, or vulnerable to, a psychotic disorder is limited. Whilst the high level of comorbid PTSD may reflect a normal reaction to a high prevalence of traumatic life events, it may be that this group exhibit an underlying cognitive processing style which makes them particularly vulnerable to the impact of a stressful life event.

A recent cognitive model of 'traumatic-psychosis' (Steel et al., 2005) suggests that a sub-group of individuals vulnerable to schizophrenia exhibit an information processing style which is associated with a high level of intrusive memories, both of past stressful events and 'everyday' neutral events. This group of individuals are particularly prone to frequent, distressing intrusive memories should they suffer a significant traumatic event. These individuals may then present with the symptoms of both schizophrenia and PTSD and are often referred to as a case of 'traumatic-psychosis'. There is some evidence of more frequent intrusive memories occurring within psychosis-prone individuals after a stressful event (Holmes and Steel, 2004). However, little is known about a possible vulnerability to more frequent neutral intrusive autobiographical memories (IAMs) within this group. The current study explored whether a group of patients suffering from comorbid schizophrenia and PTSD experienced more frequent neutral IAMs than a group of patients suffering from schizophrenia. 


\section{Method}

Individuals aged 18 to 65 with a current DSM-IV diagnosis of schizophrenia or schizoaffective disorder were recruited from mental health services within Berkshire Healthcare Foundation Trust (BHFT). Exclusion criteria were incapacity to consent, a moderate or severe learning disability and an organic origin of psychotic symptoms. Twenty-three participants completed the study, comprised of $16(70 \%)$ males and $7(30 \%)$ females with a mean age of 40.22 years $(\mathrm{SD}=8.46$, range $=27$ to 57). Specific clinical diagnosis was taken from the participant's medical records: the primary current diagnosis for 16 was of Schizophrenia and for the other 7 was Schizo-affective disorder. Anti-psychotic medications were used by 22 of the participants: other psychotropic drugs were also prescribed (anti-depressants, mood stabilizers, anti-anxiety) with a mean of 2.57 psychotropic drugs per participant $(S D=1.47)$. Traumatic events were defined using the criteria necessary for a clinical diagnosis of PTSD: "an event that involved actual death, threatened death, serious injury or threat to integrity, to themselves or others" (as per DSM-IV, APA, 1994). Trauma experience and PTSD symptoms were assessed using a revised version of the PTSD checklist, the PCL. This measure is comprised of two sections: the first listing 18 items each referring to a different category of traumatic event. Sixteen of these appear in the original form of the PCL (Weathers et al., 1993), with two additional items incorporated which are specific to psychiatric populations. These items refer to the experience of psychotic symptoms, and treatment within the psychiatric system (Tarrier et al., 2007). The second section of the PCL contains 17 items assessing a range of symptoms associated with PTSD. Participants completed this section in relation to the item endorsed in part 1 which they considered to be currently most distressing to them.

The Positive and Negative Symptom Scale for Schizophrenia (PANSS: Kay et al., 1987) was administered to assess level of current psychotic symptoms, along with the Psychotic Symptoms Rating Scale (PSYRATS: Haddock et al., 1999) in order to specifically assess delusions and hallucinations.

The procedure and stimuli used were identical to those developed by Ball (2007). A propensity to experience involuntary autobiographical memories (IAMs) was measured using a word association task in which three trials were conducted, each starting with a different cue word. A recording of the 
task was then replayed to participants, who identified whether an IAM was triggered during each trial (i.e. overall score of 0-3). Deliberate autobiographical memory (DAM) retrieval was assessed using the same three cue words as the IAM task. In each of the three trials participants were given the cue word and instructed to bring to mind an autobiographical memory relevant to that word if possible (again an overall score of 0-3). In both conditions, participants provided brief descriptions of memories retrieved and rated them for spontaneity of retrieval, amount of details retrieved and emotional valence of the event.

PCL scores were used to divide participants into two groups with scores above 44 indicating schizophrenia comorbid with PTSD. 


\section{Results}

All of the 18 items describing different types of traumatic events listed in the first section of the PCL were endorsed by at least one participant. Additionally, all participants had experienced at least one of those 18 items: the mean number of traumatic events experienced was $5.09(\mathrm{SD}=0.52)$ with a range of 1-10. Correlational analysis showed that this score of number of traumatic events experienced did not have a significant relationship with any other measure. PCL scores measuring PTSD symptomology were distributed across the full potential range of 17 to 85 with a mean score of $43.48(\mathrm{SD}=4.10)$. Ten participants scored above 44 and therefore formed the comorbid group. The comorbid group scored significantly higher than the non-comorbid group on the PANSS positive symptoms $(\mathrm{F}(1,21)=8.746, \mathrm{p}=.01, \mathrm{~d}=1.21)$ and a trends towards more severe delusions $(\mathrm{F}(1,21)=4.03, \mathrm{p}=.058, \mathrm{~d}=0.84)$ and hallucinations $(\mathrm{F}(1,21)=3.55, \mathrm{p}=.07, \mathrm{~d}=0.79)$. The comorbid group reported a higher number of traumatic events (comorbid $=6.20(\mathrm{SD}=1.62)$; non comorbid $=4.23(\mathrm{SD}=2.77))$ although the difference was not significant $(\mathrm{F}(1,20)=1.30, \mathrm{p}=.27))$.

All participants retrieved an involuntary autobiographical memory (IAM) in at least one of the three word-association trials, and in total $81 \%$ of the trials produced IAMs (see Table 1). Participants in the comorbid group produced more IAMs within the three trials than those in the non-comorbid group $(U=27.0, z=2.66, p=.02$ and $r=0.56)$. Participants were divided into high and low scoring groups based on (i) PANSS positive (ii) PSYRATS Delusions and (iii) PSYRATS Hallucinations scores. None of these groups revealed significant differences within IAM frequency.

\section{Insert Table 1 about here}

The valence of each IAM was rated on a 5-point scale: Negative (28\% of all IAMs), Slightly Negative (20\%), Neutral (27\%), Slightly Positive (19\%) or Positive (7\%). IAM valence did not differ between comorbid and non-comorbid groups $\left(\chi^{2}(4)=2.91, \mathrm{p}=.57\right)$. 
In the word-cued condition, $90 \%$ of trials led to a deliberately retrieved autobiographical memory (DAM). There was no group difference in the number of DAMs retrieved $(U=57, z=0.65, p=.52)$. People recalled more memories with deliberate retrieval than involuntarily with the word-association task but the difference was not statistically significant: $\mathrm{z}=1.90, \mathrm{p}=.09$. Memory recall was faster for IAMs than for DAMs $(\mathrm{t}(22)=7.65, \mathrm{p}<.001)$ and there was a significant difference in the participants' spontaneity rating $(\mathrm{t}(22)=6.68, \mathrm{p}=<.001)$. There was no significant difference between the two memory types in the participants' rating of the detail recalled $(\mathrm{t}(21)=0.79, \mathrm{p}=.44)$. 


\section{Discussion}

The current study is the first to explore a vulnerability to the triggering of neutral autobiographical memories within people who are diagnosed with schizophrenia and present with symptoms of PTSD. Results suggest that the comorbid group experience more frequent neutral IAMs, and that this difference is not associated with psychotic symptom severity. Further, the group difference was specific to involuntary recall and not associated with deliberate recall.

The groups did not differ in relation to the number of traumatic life events, although the severity of these events was not recorded. There was no group difference in the valence of IAM content.

One possible interpretation of the current results is, therefore, that a subgroup of individuals who are vulnerable to schizophrenia also exhibit an information processing style which makes them particularly susceptible to the impact of a stressful life event. These individuals are characterised by frequent intrusive memories of both neutral and stressful content. Should a significant stressful event occur, they are likely to experience frequent, distressing intrusive memories and symptoms consistent with a diagnosis of both schizophrenia and PTSD. However, another possibility is that the comorbid group have suffered more severe traumatic events, and that the increased vulnerability to neutral IAMs is due to a posttraumatic shift in information processing style, rather than a premorbid vulnerability factor.

With the small sample size of this study the data is not highly powered and therefore caution should be applied to any interpretation of these results. In addition, as shown in Table One, there were differences showing a higher level of IAM retrieval for participants with higher positive symptom scores (i.e. the High PANSS group) and also for participants with greater experience of hallucinations (i.e. in the High PSYRATS Hallucinations group). Whilst these differences were not statistically significant it may be that they would become so with a larger sample size and greater powered study.

A longitudinal study is required in order to inform this debate. 


\section{References}

APA, A.P.A., 1994. Diagnostic and Statistical Manual for Mental Disorders, 4th ed. APA, Washington, DC.

Ball, C.T., 2007. Can we elicit involuntary autobiographical memories in the laboratory?, in: Mace, J.H. (Ed.), Involuntary memory. Blackwell Publishing, Oxford, pp. 127-152.

Gregory, J.D., Brewin, C.R., Mansell, W., Donaldson, C., 2010. Intrusive memories and images in bipolar disorder. Behaviour Research and Therapy 48(7), 698-703.

Hackmann, A., Clark, D.M., McManus, F., 2000. Recurrent images and early memories in social phobia. Behaviour Research and Therapy 38(6), 601-610.

Haddock, G., Mccarron, J., Tarrier, N., Faragher, E.B., 1999. Scales to measure dimensions of hallucinations and delusions: the psychotic symptom rating scales (PSYRATS). Psychological Medicine 29, 879-889.

Holmes, E.A., Steel, C., 2004. Schizotypy: A Vulnerability Factor for Traumatic Intrusions. Journal of Nervous and Mental Disease 192(1), 28-34.

Kay, S.R., Flszbein, A., Opfer, L.A., 1987. The Positive and Negative Syndrome Scale (PANSS) for Schizophrenia. Schizophrenia Bulletin 13(2), 261-276.

Kilcommons, A.M., Morrison, A.P., 2005. Relationships between trauma and psychosis: an exploration of cognitive and dissociative factors. Acta Psychiatrica Scandanavia 112, 351-359.

Spauwen, J., Krabbendam, L., Lieb, R., Wittchen, H.-U., van Os, J., 2006. Impact of psychological trauma on the development of psychotic symptoms: relationship with psychosis proneness. British Journal Of Psychiatry 18(8), 527-533.

Speckens, A.E.M., Hackmann, A., Ehlers, A., Cuthbert, B., 2007. Imagery special issue: Intrusive images and memories of earlier adverse events in patients with obsessive compulsive disorder. Journal of Behavior Therapy and Experimental Psychiatry 38(4), 411-422.

Steel, C., Fowler, D., Holmes, E.A., 2005. Trauma-Related Intrusions and Psychosis: An Information Processing Account. Behavioural and Cognitive Psychotherapy 33(2), 139-152.

Tarrier, N., Khan, S., Cater, J., Picken, A., 2007. The subjective consequences of suffering a first episode psychosis: Trauma and suicide behaviour. Social Psychiatry and Psychiatric Epidemiology 42(1), 29-35.

Weathers, F.W., Litz, B.T., Herman, D.S., Huska, J.A., Keane, T.M., 1993. The PTSD checklist: reliability, validity, \& diagnostic utility, Annual Meeting of the International Society for Traumatic Stress Studies, San Antonio, TX, October. 
Table One: Number of word-association trials eliciting an IAM, by group

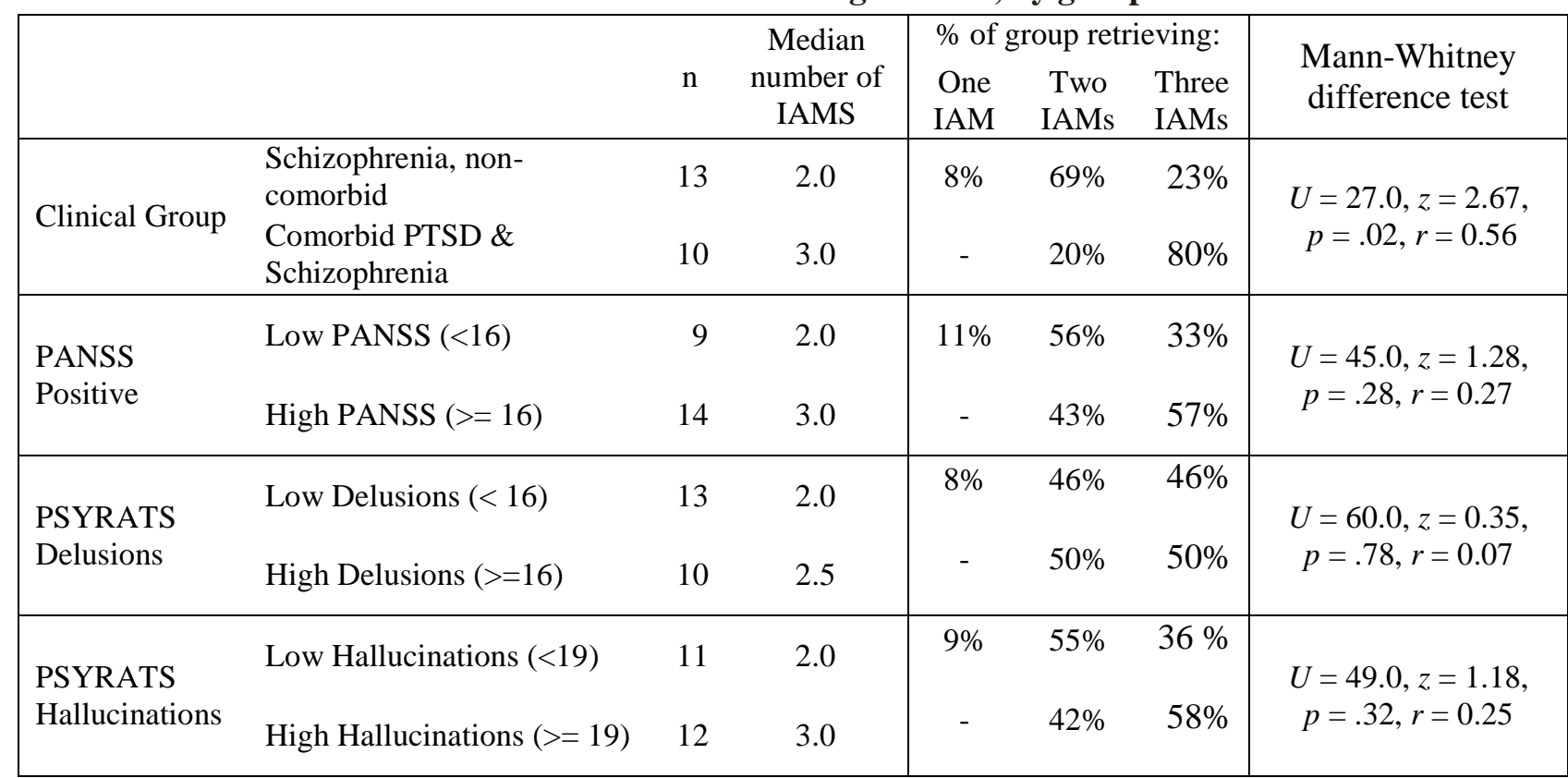

